\title{
Thick metal plate insertion make FR4 multilayer board a simple carrier for RF power circuits
}

\author{
Carlo Buoli , Giovanni Biffi, Tommaso Turillo, Alessandro Zingirian
}

Siemens Information and Communication Networks S.p.A., Strada Padana Superiore Km158 20060 ,

Cassina de Pecchi - MILANO.

e-mail: Carlo.Buoli@icn.siemens.it, Giovanni_Ivano.Biffi@icn.siemens.it,Tommaso.Turillo@icn.siemens.it, Alessandro.Zingirian@icn.siemens.it

\begin{abstract}
In order to reduce sizes and costs of microwave circuits and to simplify their integration together with IF, control and power supply networks, we tested (1) a simple solution that allows using FR4 boards also for microwave circuits. Since FR4 boards are necessarily present in every equipment for power supplies, control, digital and IF circuits, it becomes possible to reduce the number of boards or modules collecting all the networks (operating from DC to tenth of Gigahertz) on the same FR4 multilayer.

The typical RF requirements of good thermal and electrical ground and the need of a carrier for "chip on board" monolithic assembly are all achieved through the insertion of a copper plate a few tenth of millimetre thick. This copper plate replaces the second metal layer of FR4 multilayer remaining within standard FR4 process; monolithics can be mounted on it just opening a window on the first layer.
\end{abstract}

\section{INTRODUCTION}

In this paper we present a multilayer structure that allows us to integrate microwave circuits together with IF, control and power supply circuits.

This structure is simple and low-cost since it can be obtained using the standard processes. The peculiarity consists in the insertion of a thick copper whose function is electrical ground, thermal ground and mechanical support.

Furthermore there are not intrinsic limitation on operating frequency because a quasi-TEM propagation in a microstrip is assured when the wavelength is much greater than width. Our structure can easily satisfy this condition. Some problems have been found considering losses: while on alumina they are practically negligible, on FR4 they are approximately $1 \mathrm{~dB}$ per wavelength (2); nevertheless in many applications these difficulties can be overcome giving an important weight to minimisation of layout dimension since the design first steps.

\section{THE STRUCTURE}

In Figure 1 the suggested structure is schematically shown: it is a 4-layer FR4 printed circuit board: microstrips are on the first layer whose dielectric is 100 micron thick. On this layer, 200-micron width track is a nearly 50 -ohm impedance microstrip, and quasi-TEM propagation can be obtained up to $80 \mathrm{GHz}$, since at this frequency the wavelength-to-width ratio is about 10 , which is yet a reasonable value.

The second metal layer is a copper plate $0.5 \mathrm{~mm}$ thick, and it is both electrical and thermal ground for microwave devices and offers a strong support to the upper thin FR4 layer.

The third layer can house supply and control circuits.

The fourth layer is a further ground plane, generally used as electrical ground for DC and low frequency circuits.

\section{DESIGN APPROCHES}

Alumina inserts - If low losses are a need, it is possible to build circuits on alumina (or other hi-Q substrate) and mount them directly on the copper plate through a suitable window opened on the first metal and dielectric layer.

Chip on board - The previous procedure can be also used for chip mounting, taking advantage of the good electrical and thermal grounding of the thick copper plate. To this purpose, the upper dielectric layer thickness must be as closer as possible to the chip one, so that the bonds are shorter minimising their 
parasitic effects. Note that dielectric thickness affects microstrip impedance so we choose 100 micron, the lowest value to achieve $50 \mathrm{ohm}$ impedance remaining within the FR4 multilayer standard process. The monolithics and their bonds can be covered with an epoxy glob top in order to protect them.

Isolators - We can likewise integrate isolators on the same board: in the slot opened on the first layer we will insert a garnet disk, and a magnet will be glued onto it.

Multilayer circuits - Bias circuits and low frequency networks can take place on the third layer. In this way it is easier to connect, on the whole board, the several devices bias point and to complete the layout without interfering with microwave regions that cannot be modified without troubles.

Transition microstrip-guide - Guide transitions are feasible too, etching the base material under the copper plate and screwing the board to a metal housing that incorporate the waveguide (see Figure 2); in this way the copper plate could even replace the upper guide boundary.

Packaging - It is pointed out that by using this kind of microwave structure the packaging can be easily designed. In fact the multilayer itself may behave like a box if the RF-circuits are surrounded by a series of plate holes distant approximately one eighth of wavelength each other (see Figure 3).

Consider also that FR4 losses reduce risks of self-oscillations, since their behaviour is similar to eccosorb sheets that are often glued on the box covers and walls.

\section{PRACTICAL IMPLEMENTATION AND RESULTS}

We experimentally verified all the possibilities offered by the structure just described.

Particularly we tested chip-on-board, mounting a broadband MMIC amplifier and testing it in the $20-27 \mathrm{GHz}$ band (see Figure 3). We measured a 16dB flat gain all over the band (see Figure 4). The circuit absorbs 240mA from the 5V supply: we tested, over a several hours period, that the thermal dissipation was good.

Then we designed a microstrip-to-waveguide transition (3) and simulated it using Agilent HFSS. The model for simulation is described in Figure 5. The slot-line and the step impedance transformer are obtained in the thick copper plate; the waveguide is included in the metal housing so that the board will be screwed up to it. Simulation has been performed taking into account, for FR4, a dielectric constant of 4.6 and a dielectric loss tangent of 0.025. Results are in Figure 6 and show an insertion loss not exceeding 0.5dB. These results led us to build a whole transmitter in the $24.5-26.5 \mathrm{GHz}$ band (Figure 7). In Figure 8 is shown the final stage that uses a medium-power MMIC amplifier. We measured $28 \mathrm{dBm}$ output power at $1 \mathrm{~dB}$ compression and the relative frequency response is rather flat over the whole band of interest (Figure 9). Two-tone output spectrum shows intermodulation products level lower than $-45 \mathrm{dBc}$ (Figure 10). We protected all monolithics covering them with epoxy glob top verifying an output power degradation of $0.3 \mathrm{~dB}$.

\section{CONCLUSIONS}

Suitable cautions in the design and the insertion of a copper plate as a second layer in an FR4-multilayer allow easily achieving:

1) the integration of both low and high frequency circuits on the same board exploiting simple standard FR4 technology;

2) automatic mounting of monolithics or circuits that need to be built on a different substrate (i.e. alumina) because of their low loss requirements or critical tolerances;

3) high power dissipation, by suitably dimensioning the copper plate thickness.

All that turns out into closer structures. As a consequence costs and assembly time are considerably reduced, in comparison with the usual hybrid technology.

\section{REFERENCES}

(1) Patent pending

(2) C. Buoli, G. Mora, L.A. Cervi; FR4 PCB Modulation Transfer MWVCO Up to 16 GHz; Sept.1997; 27th European Microwave Conf., Jerusalem, Israel.

(3) J.F. Zürcher , F.E. Gardiol; Broadband Patch Antennas; 1995; Artech House; chapter 2, pp.19-41 


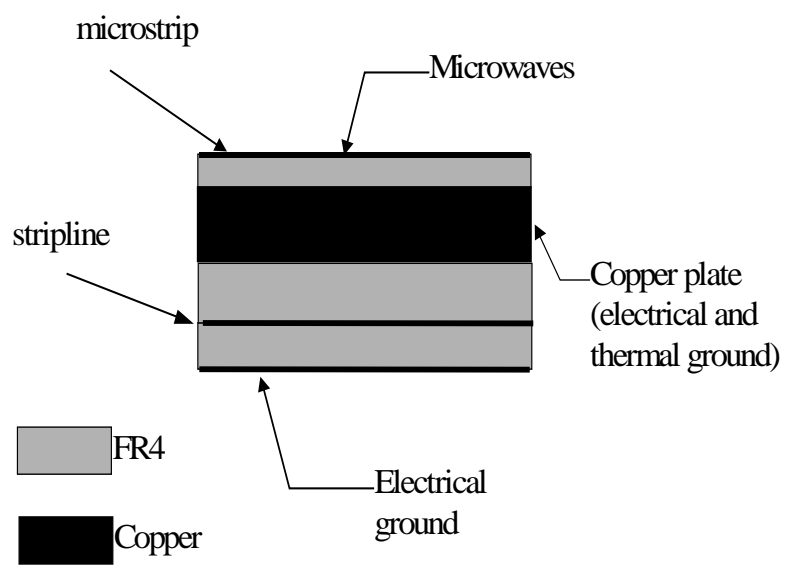

Figure 1 - The multilayer structure with copper plate inside

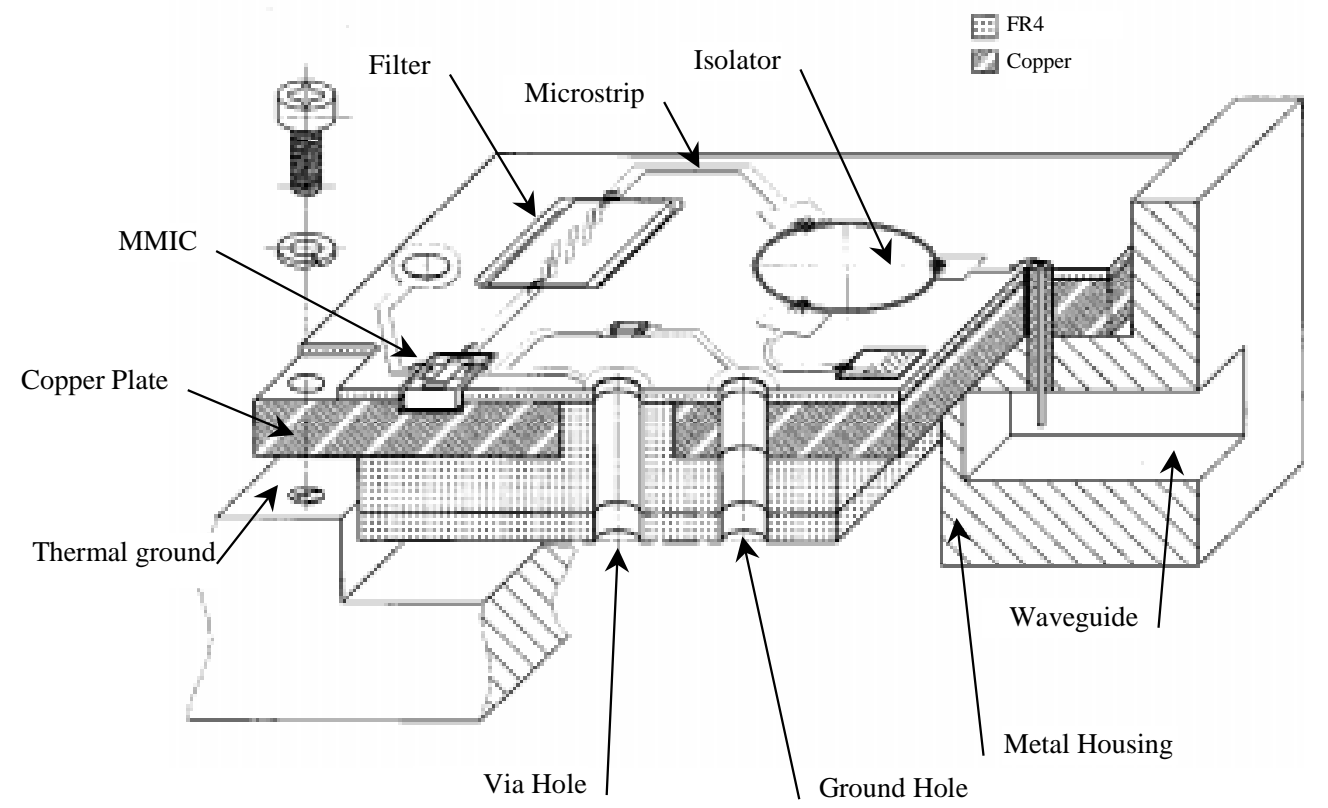

Figure 2 - Alumina filters, monolithics, isolators, guide transitions on the proposed structure.

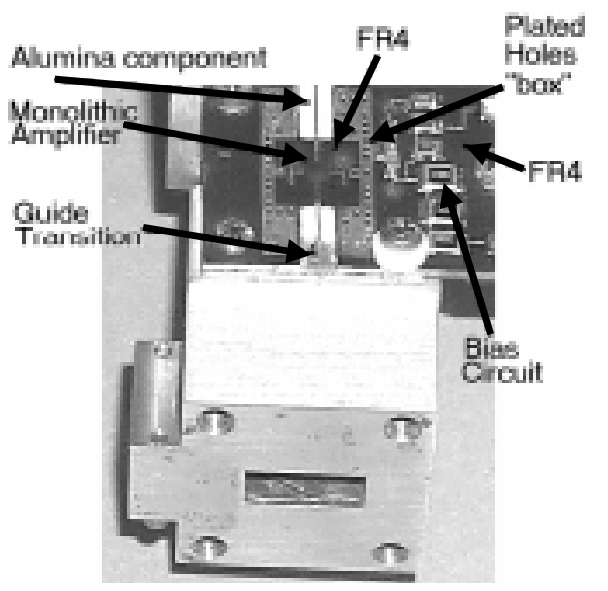

Figure 3 - Broadband power amplifier practical implementation $(\mathbf{P 1 d B}=\mathbf{2 0 d B m})$.

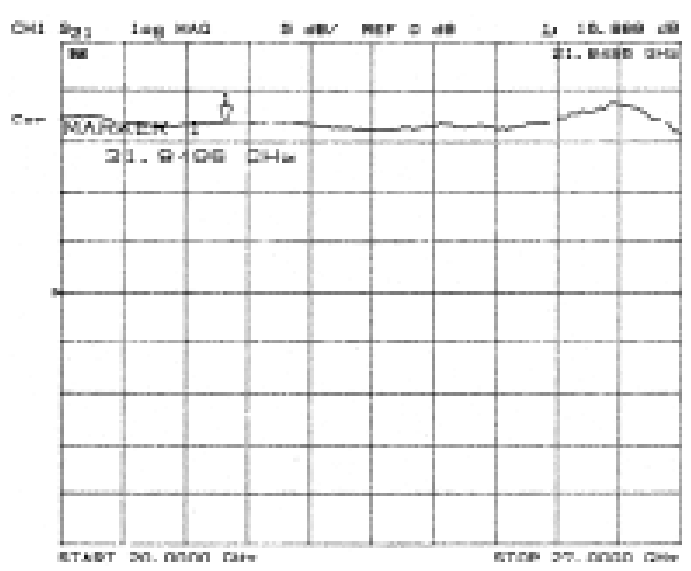

Figure 4 - Broadband power amplifier measurement. 


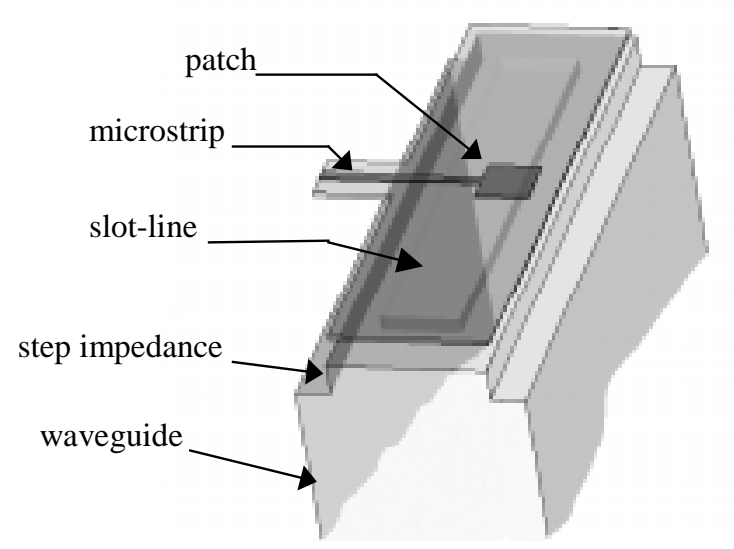

Figure 5 - Model of the microstrip-to-waveguide transition for electromagnetic simulation.

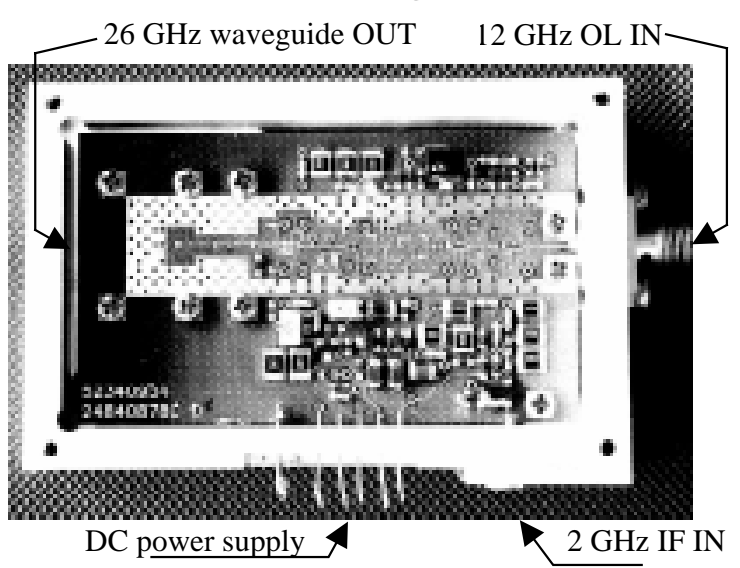

Figure 7 - The whole transmitter structure.

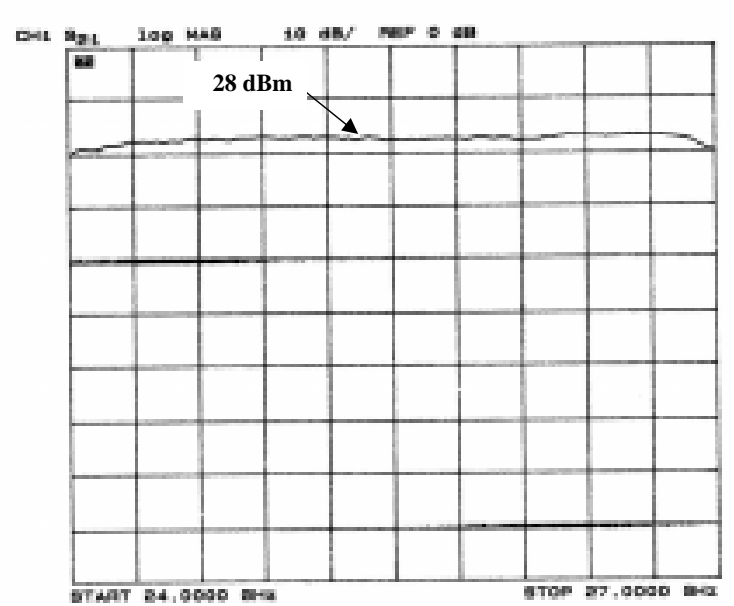

Figure 9 - Transmitter output power measurement.

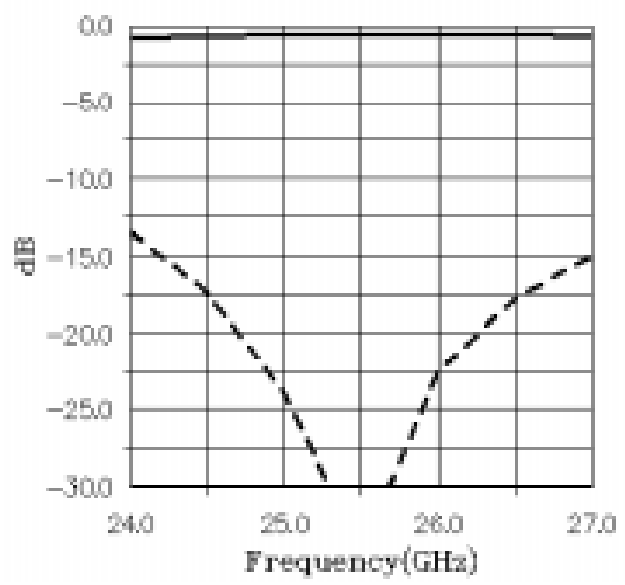

Figure 6 - Microstrip-to-waveguide transition HFSS simulation results.

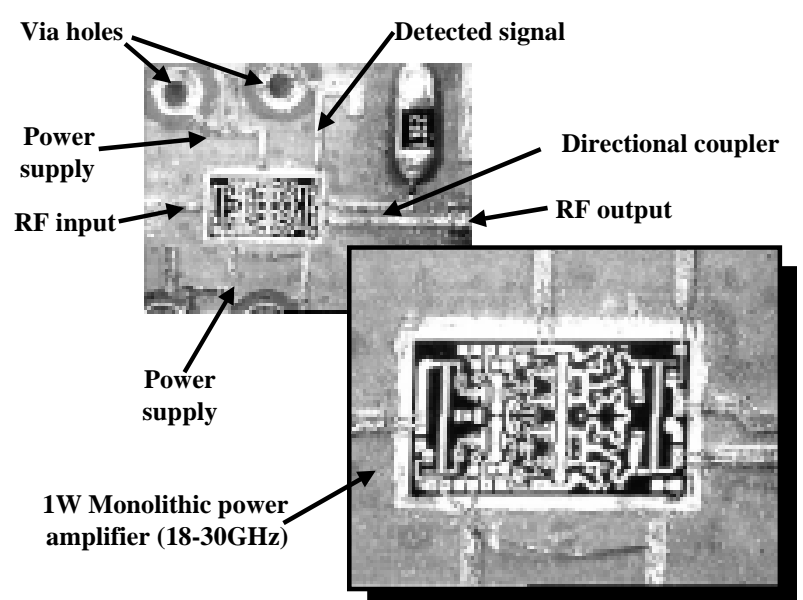

Figure 8 - "Chip on board” on FR4 multilayer.

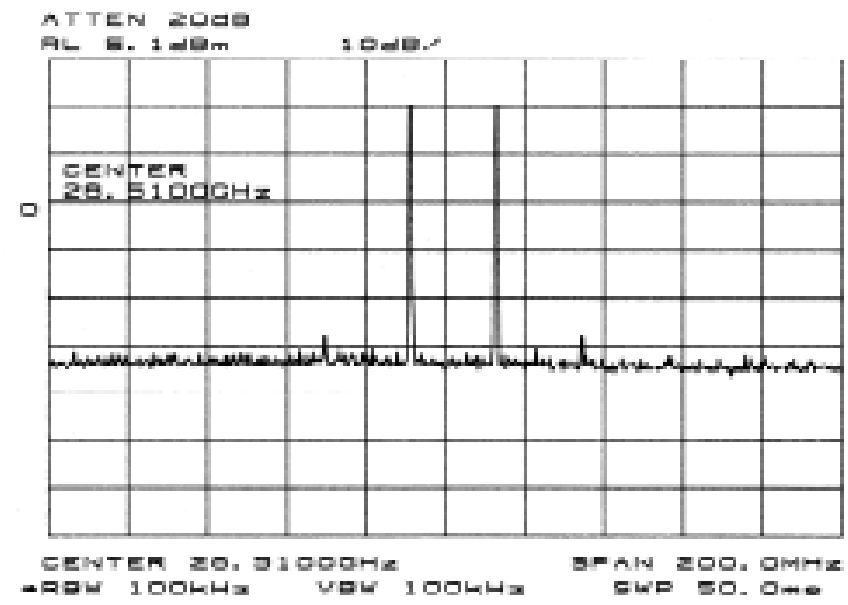

Figure 10 - Two tone RF output signal spectrum (Pout=16dBm single carrier level). 